\title{
Psychometric validation of a knowledge questionnaire on micronutrients among adolescents and its relationship to micronutrient status of 15-19-year-old adolescent boys, Hyderabad, India
}

\author{
Little Flower Augustine ${ }^{1}$, Shahnaz Vazir ${ }^{2}$, Sylvia Fernandez Rao ${ }^{2}, M$ Vishnuvardhana Rao ${ }^{3}$, \\ A Laxmaiah ${ }^{4}, \mathrm{P}_{\text {Ravinder }}{ }^{1}$, V Vikas Rao ${ }^{1}$ and K Madhavan Nair ${ }^{1, *}$ \\ 'Division of Micronutrient Research, National Institute of Nutrition, Indian Council of Medical Research, \\ Jamai-Osmania, Hyderabad 500007, Andhra Pradesh, India: ${ }^{2}$ Division of Behavioral Sciences, National \\ Institute of Nutrition, Indian Council of Medical Research, Hyderabad, India: ${ }^{3}$ Division of Biostatistics, National \\ Institute of Nutrition, Indian Council of Medical Research, Hyderabad, India: ${ }^{4}$ Division of Community Studies, \\ National Institute of Nutrition, Indian Council of Medical Research, Hyderabad, India
}

Submitted 23 June 2011: Accepted 19 December 2011: First published online 10 February 2012

\begin{abstract}
Objective: To develop a psychometrically valid questionnaire for testing knowledge on micronutrients and to assess the relationship between knowledge and biomarkers of micronutrient status among adolescents.

Design: Cross-sectional, institution-based, validity and reliability study.

Setting: Seven higher secondary schools were covered in the limits of Greater Hyderabad Municipal Corporation, Hyderabad, India.

Subjects: Students aged 15-19 years, $n 92$ for the pre-test, $n 108$ for test-retest and $n 109$ for studying the relationship between knowledge and biomarkers of Fe, retinol, ascorbic acid, $\alpha$-tocopherol, folic acid and vitamin $B_{12}$ status.

Results: From an item pool of 106, thirty-one items were selected based on content validity. Statistical tools to obtain a valid and reliable questionnaire among adolescent boys and girls resulted in eighteen items with a difficulty index of $0 \cdot 11-0 \cdot 86$, discrimination index of $0 \cdot 20-0 \cdot 72$ and validity index (point bi-serial correlation) of $0 \cdot 10-0 \cdot 62$. Reliability as measured by Cronbach's $\alpha$ was $0 \cdot 71$ and the intra-class correlation coefficient was $0 \cdot 80$. A Bland-Altman plot showed good agreement between test and retest scores. The mean response score to the eighteen-item questionnaire was $5 \cdot 2$ (sD $2 \cdot 68$ ). The mean values of serum retinol were significantly different $(P=0 \cdot 022)$ between groups below $(24 \cdot 8(\mathrm{sD} 6 \cdot 64) \mu \mathrm{g} / \mathrm{dl})$ and above $(28 \cdot 0$ (sD 7.67) $\mu \mathrm{g} / \mathrm{dl}$ ) the 50 th percentile of knowledge score. The relationship persisted after controlling for economic status as a covariate using analysis of covariance $(P=0 \cdot 018)$. Other micronutrients did not show any significant relationship.

Conclusions: A valid and reliable eighteen-item knowledge questionnaire was constructed and found to have a significant positive relationship with plasma retinol status alone.
\end{abstract}

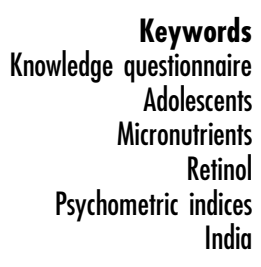

Micronutrient deficiencies are rampant in India among all age groups. The intervention strategies include supplementation, fortification and dietary diversification, the latter being the most sustainable approach. This strategy can be promoted through intense education and mass awareness programmes. Lack of culture-appropriate nutrition education has been identified as one of the weakest links of nutrition interventions in India targeting micronutrient malnutrition ${ }^{(1)}$. One of the obstacles for this could be the lack of information on current nutrition knowledge among different population groups, which in turn can be attributed to the lack of validated assessment tools for testing knowledge among target groups. The need for valid and reliable tools in nutrition education research for assessment of knowledge is now well recognized on account of the realization that the poor association between nutritional knowledge and dietary intake may be due to the poor assessment of knowledge ${ }^{(2-12)}$.

Development of a valid questionnaire based on predefined criteria was suggested by Kline ${ }^{(2)}$. Applying these criteria, a general nutrition knowledge questionnaire was 
developed for the US adult population which has been translated, pre-tested and used widely ${ }^{(3-5)}$. Attempts have been made elsewhere to develop reliable questionnaires for nutrition education research, focusing on general nutrition knowledge of adults ${ }^{(6,7)}$. Tools have also been developed for use among elementary-school teachers and children aimed at measuring the dietary intake or general nutrition knowledge ${ }^{(8-10)}$. Additionally, there have been needs-based attempts in the past to develop questionnaires addressing specific areas of nutrition knowledge like fat, fibre and cholesterol ${ }^{(10,11)}$. A questionnaire was also developed for assessment of physical activity, diet, weight-related attitudes and cultural identity among American Indian children ${ }^{(12)}$.

Furthermore, studies on the relationship between nutrition knowledge and nutritional status are often restricted to either intake data or body weight ${ }^{(13-15)}$. Studies attempting to relate nutrition knowledge to the biomarkers of nutrients are difficult to find. Such information can however be helpful for strengthening validity in the development of nutrition knowledge questionnaires. A valid and reliable questionnaire can help nutrition scientists to monitor the impact of interventions aimed at improving the dietary intake of micronutrients.

There are no validated tools in the literature to assess knowledge on micronutrients, deficiency of which is an emerging area of concern in developing countries like India. Since adolescence is an age group where maximum behaviour change is possible ${ }^{(16)}$, the aim of the present study was to develop a psychometrically valid and reliable questionnaire for testing knowledge on micronutrients. The study also attempted to apply the validated questionnaire among a group of adolescents whose micronutrient status was already assessed, to understand the relationship between knowledge and biomarkers of micronutrient status.

\section{Methods}

All procedures and protocols were approved by the Institutional Ethics Committee. Permission was obtained from the principals of the participating schools and the Directorate of Intermediate Education, Government of Andhra Pradesh - a southern state of India. The study was carried out in 2009-2010 in Government-run schools which cater to students belonging to low- and middleincome families. A written informed consent from parents, and assent from students, was obtained.

\section{Development of questionnaire}

The questionnaire was constructed using the method suggested by Kline ${ }^{(2)}$ and Garret and Woodworth ${ }^{(17)}$. The process of questionnaire construction consisted of forming an item pool, carrying out a content analysis, and analysing the item difficulty, discriminative power, validity and reliability.

\section{Item pool}

The item pool addressed specific micronutrients on the basis of: (i) those that were known to be deficient among the population; (ii) those that had a reliable biomarker; and (iii) intake data being available. Among the fat-soluble vitamins, vitamins A and E were selected. Vitamin C, vitamin $\mathrm{B}_{12}$ and folic acid were addressed among the water-soluble vitamins. Fe was the only mineral considered on account of its low intake and widespread deficiency. The questions were designed within three major domains: (i) functions; (ii) signs of deficiencies; and (iii) sources of the micronutrients. A few questions on general health, nutrition and national programmes were also included (Table 1). An item pool of 106 questions was constructed from the literature, available validated questionnaires and by consulting experts in the field of nutrition. The item pool was subjected to content analysis.

Table 1 Knowledge segments covered under the domains of function, deficiency and food sources in the item pool

\begin{tabular}{|c|c|c|}
\hline No. & Segment & No. of items \\
\hline 1. & $\begin{array}{l}\text { Micronutrient deficiency } \\
\text { Causal relationship: anaemia, night blindness and scurvy } \\
\text { Emerging evidence: non-communicable diseases and role in common infections like cold }\end{array}$ & 27 \\
\hline 2. & $\begin{array}{l}\text { Awareness on nutrients and nutrition } \\
\text { Balanced diet and ability to identify food sources of carbohydrates, protein, fat, vitamins and minerals } \\
\text { in the diet }\end{array}$ & 23 \\
\hline 3. & $\begin{array}{l}\text { Food sources of micronutrients } \\
\mathrm{Fe} \text {, vitamin } \mathrm{A} \text {, vitamin } \mathrm{C} \text {, folic acid, } \mathrm{B} \text { vitamins, vitamin } \mathrm{E} \text { and vitamin } \mathrm{B}_{12}\end{array}$ & 21 \\
\hline 4. & $\begin{array}{l}\text { Micronutrient functions } \\
\text { Maintaining general health and vigour, synthesis of } \mathrm{Hb} \text {, role in cognition, brain development, work capacity, } \\
\text { attention concentration and depression }\end{array}$ & 11 \\
\hline 5. & $\begin{array}{l}\text { Nutrient availability and storage in the body } \\
\text { Emphasis on absorption promoters and inhibitors, storage of fat-soluble and water-soluble vitamins }\end{array}$ & 13 \\
\hline 6. & $\begin{array}{l}\text { Processing losses } \\
\text { Washing prior to cooking and cooking per se }\end{array}$ & 6 \\
\hline 7. & $\begin{array}{l}\text { Awareness on hygiene } \\
\text { Hand washing and worm infestation }\end{array}$ & 2 \\
\hline 8. & $\begin{array}{l}\text { Awareness on national programmes } \\
\text { Addressing micronutrient deficiencies - vitamin A, Fe, folic acid and iodine } \\
\text { Total number of items }\end{array}$ & $\begin{array}{r}3 \\
106\end{array}$ \\
\hline
\end{tabular}




\section{Content analysis}

Content analysis was done by the judgement method. The questions were direct and no negative phrasing was used. Two independent reviews were carried out by a panel of five experts from the fields of nutrition, psychology, medicine and basic sciences to select the questions that were appropriate, accurate and interpretable. The acceptance, rejection or modification of an item was based on majority opinion.

\section{Pre-test}

The questionnaire was translated to the regional language, Telugu, using the forward-backward translation method and pre-tested in a group of adolescents aged 15-19 years studying in 12th grade. The questionnaire was selfadministered. The group consisted of equal number of students from science and commerce streams ( $n$ 92) from both genders (thirty-seven girls and fifty-five boys).

\section{Scoring}

The thirty-one-item questionnaire consisted of twenty-eight closed-end questions and three open-ended questions. The closed-end questions consisted of sixteen 'yes/no' questions and twelve multiple-choice questions. There was only one correct answer to each question and an option of 'not sure' was used to reduce response bias. The responses were scored by awarding a mark of 1 to the correct response and a mark of 0 to an incorrect or blank response.

\section{Item difficulty index}

The item difficulty index $(p)$ was determined by the number of students in the group who performed correctly on the item and expressed as a ratio of correct responses to the total number of questions. An item with a value of $p$ below $0 \cdot 20$ was considered difficult, while $p$ above $0 \cdot 80$ indicated an easy item ${ }^{(17)}$.

\section{Item discrimination index}

The steps involved in determining the item discrimination index $(D)$ consisted of identifying the $27 \%$ of students who had the highest overall scores and the $27 \%$ with the lowest scores, representing the tail from the mean of the standard normal distribution of response error ${ }^{(17)}$. The $p$ levels for the upper and lower groups were determined as described above, and then the $p$ levels of the lower group were subtracted from those of the upper group; the resulting values were considered as $D$. The higher the $D$, the more discriminating was the item. A value of $D=0 \cdot 20-0 \cdot 29$ was considered 'acceptable', $D=0 \cdot 30-0 \cdot 39$ as 'good' and $D>0 \cdot 40$ was considered 'excellent' as per available literature $^{(18)}$.

\section{Item validity index}

The validity index of each item (calculated as point bi-serial $r$ ) was determined by the extent to which the given item could discriminate among students who differed sharply in the function measured by the test as a whole and was determined by the inter-correlation between each item in a scale ${ }^{(7)}$. Point bi-serial $r$ is mathematically equivalent to Pearson product moment correlation and is used when one of the variables is dichotomous. A point bi-serial $r$ of $0 \cdot 2$ was considered valid ${ }^{(2)}$ except in circumstances where an item was considered essential in terms of content validity. In such cases, an $r$ value of $\geq 0 \cdot 1$ was considered a minimum requirement as described in the literature ${ }^{(17)}$.

\section{Internal consistency}

The rational equivalence method using Cronbach's $\alpha$ was employed to assess the reliability of the test. Cronbach's $\alpha$ estimates the reliability of test scores with respect to (i) how well the individual items of the scores fit together and (ii) whether they assess the same construct. It assesses the inter-correlations of the items in the test and the correlations of the items with the test as a whole. A value of $\alpha$ above 0.70 was considered acceptable on the internal consistency as per available literature ${ }^{(2,19)}$ and was done using the SPSS statistical software package version $16 \cdot 0$ (SPSS Inc., Chicago, IL, USA).

\section{Test-retest reliability}

Considering a minimum of five observations for each item and a drop-out rate of $20 \%$, in total 108 students were considered sufficient for the study on test-retest reliability of the eighteen-item questionnaire that was developed. The questionnaire was self-administered. The time taken to complete the questionnaire was noted down using a wrist watch. The students were kept unaware of the retest that was done after a gap of $7 \mathrm{~d}^{(9)}$. A total of 100 students completed both the contacts of the test-retest. A Bland-Altman plot was constructed and the intra-class correlation coefficient (ICC) was calculated for assessing the agreement between the scores ${ }^{(20,21)}$. An ICC above $0 \cdot 75$ was considered acceptable ${ }^{(21)}$.

\section{Relationship between biomarkers and knowledge on micronutrients}

The knowledge questionnaire was applied to a group of students participating in a 2-year study on 'Stress, allostatic load and micronutrient status among students: impact of dietary advice'. For this, five single-gender schools catering to male students from three different geographical locations of Greater Hyderabad Municipal Corporation, Andhra Pradesh, India were selected. Students with congenital anomalies, physical handicaps or any other chronic health problems requiring medical attention and those taking micronutrient tablets for the past year were excluded from the study. A total of 380 students were recruited for the study using probability proportional to size sampling, from whom a blood sample was collected for analysis of micronutrient status in a randomly selected sub-sample of 150 . One hundred 
and nine of these participants were available for testing the validated knowledge questionnaire, which was done after a gap of 6 months.

\section{Micronutrient status}

The baseline blood samples were collected after an overnight fast and analysed for $\mathrm{Hb}^{(22)}$ and plasma ferritin ${ }^{(23)}$, folic acid and vitamin $B_{12}$ (RIA kit by Siemens Inc., LA, USA). Simultaneous determination of plasma retinol and $\alpha$-tocopherol was carried out by HPLC (Thermo Finnigan, Herts, UK) ${ }^{(24)}$. An ascorbic acid assay was done on the same day using the $\alpha, \alpha$-bipyridyl micromethod ${ }^{(25)}$. The ferritin ELISA and HPLC methods for retinol and $\alpha$-tocopherol were in-house methods and are continuously validated by the VITAL-EQA (Vitamin A Laboratory - External Quality Assurance) programme of the Centers for Disease Control and Prevention (Atlanta, GA, USA). The percentage inadequacy of micronutrient status was computed using cut-off values derived from the literature ${ }^{(26)}$.

\section{Economic status}

Economic status was derived from the information collected on twelve household assets by computing the standard of living index ${ }^{(27)}$.

\section{Statistical analysis}

All statistical analyses were done using SPSS version $16 \cdot 0$. Descriptive statistics were used to summarize the results. Item analysis (item difficulty index $p$, item discrimination index $D$, point bi-serial $r$ and Cronbach's $\alpha$ ) was done for psychometric validation of the thirty-one-item questionnaire. The Bland-Altman plot was constructed using the MedCalc statistical program version $11 \cdot 5 \cdot 1 \cdot 0$ (MedCalc Software, Mariakerke, Belgium). The relationship between knowledge and biomarkers of micronutrient status was tested by the two-tailed independent-samples $t$ test between groups above and below the 50th percentile of knowledge score. For micronutrients which were not normally distributed, the non-parametric Mann-Whitney $U$ test was used. Analysis of covariance was performed taking the standard of living index as a covariate, knowledge as the independent variable and micronutrient status as the dependent variable.

\section{Results}

The content analysis of the 106 items resulted in the selection of six multiple-choice questions, twenty-one yes/no questions and four one-word-answer type questions. There was no significant gender difference in the scores of respondents during the pre-test $(P=0.995)$ and therefore only pooled analysis was done.

\section{Psychometric indices}

The item difficulty index $p$ ranged from $0 \cdot 11$ to $0 \cdot 86$. There were three relatively easy questions $(p=0 \cdot 84-0 \cdot 86)$ and three difficult questions $(p=0 \cdot 11-0 \cdot 19)$ and all the rest were in the desirable range of difficulty (Table 2 ).

The item discriminative index $D$ ranged from -0.32 to $0 \cdot 72$. Eight items with a discriminative index below $0 \cdot 20$ were discarded as poorly discriminating questions (Table 2 ).

Twelve questions had item validity index $r$ above $0 \cdot 2$. Due to good content validity, six items in the range of $r$ from $0 \cdot 1$ to $0 \cdot 2$ were retained, resulting in an eighteenitem questionnaire (Table 3 ).

A Cronbach's $\alpha$ value of 0.71 was obtained for the eighteen-item scale. The domains were combined in the shortened version. The Bland-Altman plot showed a mean of 0.8 with few outliers (Fig. 1). The ICC of $0 \cdot 80$ was significant at $P<0 \cdot 01$. The final questionnaire consisted of twelve items on functions/deficiencies, five items on dietary source and one question on hand washing (hygiene). The questionnaire took about $15 \mathrm{~min}$ to complete.

\section{Relationship between the valid eighteen-item questionnaire and micronutrient status}

The mean age of the students was $16 \cdot 6$ (SD 1.24) years; their mean height was $163 \cdot 7(\mathrm{sD} 6.95) \mathrm{cm}$, mean weight was $47 \cdot 5$ (SD $7 \cdot 37) \mathrm{kg}$ and mean BMI was $17 \cdot 7$ (sD $2 \cdot 23) \mathrm{kg} / \mathrm{m}^{2}$.

The knowledge scores of the students ranged from 0 to 14 , with a mean of $5 \cdot 2$ (SD $2 \cdot 68$ ). Fifty-nine per cent of the students scored below the 50th percentile of knowledge score, i.e. a score of less than $5 \cdot 0$.

\section{Micronutrient status}

The mean values of micronutrients were 13.9 (sD 1.63) g/dl for $\mathrm{Hb}, 44 \cdot 6(\mathrm{sD} 80 \cdot 1) \mathrm{ng} / \mathrm{ml}$ for ferritin, $0 \cdot 42(\mathrm{sD} 0 \cdot 29) \mathrm{mg} / \mathrm{dl}$ for ascorbic acid, $26 \cdot 3(\mathrm{sD} 7 \cdot 4) \mu \mathrm{g} / \mathrm{dl}$ for plasma retinol, 328.9 (sD 164.42) $\mu \mathrm{g} / \mathrm{dl}$ for $\alpha$-tocopherol, $5 \cdot 5$ (sD 1.9 ) $\mathrm{ng} / \mathrm{ml}$ for folic acid and $246 \cdot 0$ (SD 110.00) pg/ml for vitamin $\mathrm{B}_{12}$.

\section{Knowledge and micronutrient status among students}

Plasma retinol status was significantly different between the groups below and above the 50th percentile of knowledge score $(P=0 \cdot 022)$. Other biomarkers of micronutrient status did not show any significant relationship with knowledge score (Table 4). The analysis of covariance performed with micronutrient status as the dependent variable and standard of living index as a covariate also confirmed a significant difference in retinol status based on knowledge score $(P=0 \cdot 018)$.

\section{Discussion}

Several studies assessing knowledge have been found to report on a questionnaire being 'pre-tested' without providing the statistical attributes that establish its reliability and validity ${ }^{(28-31)}$. A structured approach of scaling is of prime importance for the construction of a knowledge questionnaire in order to establish its validity. To our understanding, there are no validated questionnaires 
Table 2 Difficulty index and discrimination index values of questions in the knowledge questionnaire

\begin{tabular}{|c|c|c|c|c|c|c|c|}
\hline No. & Item & $p$ & $D$ & No. & Item & $p$ & $D$ \\
\hline 1. & Example of a vitamin & $0 \cdot 61$ & $0 \cdot 24$ & 16. & Animal foods as the major food source of vitamin $B_{12}$ & $0 \cdot 30$ & 0.32 \\
\hline 2. & Iron as an essential mineral for the body & $0 \cdot 86$ & 0.32 & 17. & Vitamin $\mathrm{C}$ as the absorption promoter of iront & $0 \cdot 44$ & 0.24 \\
\hline 3. & Low intake of iron as a reason for anaemia & 0.57 & 0.60 & 18. & Groundnuts as a source of vitamin E & 0.51 & $0 \cdot 04^{*}$ \\
\hline 4. & Less activity exhibited by anaemic & $0 \cdot 39$ & $0.04^{*}$ & 19. & Fruits \& vegetables as a source of $B$ vitamins & $0 \cdot 74$ & $-0 \cdot 32^{*}$ \\
\hline 5. & Iron as an integral part of haemoglobin & $0 \cdot 41$ & 0.72 & 20. & Washing hands for prevention of infections (iron) & 0.47 & 0.56 \\
\hline 6. & Familiarity on folic acid & $0 \cdot 84$ & $0 \cdot 20$ & 21. & Beta-carotene as pro-vitamin At & $0 \cdot 39$ & 0.24 \\
\hline 7. & Role of folic acid in red blood cell formation & $0 \cdot 39$ & $0 \cdot 24$ & 22. & Colour code for beta-carotene & $0 \cdot 20$ & $0 \cdot 08^{\star}$ \\
\hline 8. & Vitamin A deficiency as the cause for night blindness & 0.45 & 0.28 & 23. & Lime as a good source of vitamin C & $0 \cdot 68$ & $0.08^{*}$ \\
\hline 9. & Familiarity with 'fat-soluble vitamins' & $0 \cdot 75$ & 0.32 & 24. & Cooking losses of B vitaminst & 0.43 & $0 \cdot 20$ \\
\hline 10. & Example of a fat-soluble vitamin & $0 \cdot 22$ & 0.60 & 25. & Fresh vegetables \& fruits as source of B vitamins & $0 \cdot 79$ & $0.04^{*}$ \\
\hline 11. & Familiarity with 'water-soluble vitamins' & $0 \cdot 37$ & 0.36 & 26. & Cooking loss of vitamin C & $0 \cdot 30$ & 0.44 \\
\hline 12. & Example of a water-soluble vitamin & $0 \cdot 19$ & 0.52 & 27. & Banana as an easily available source of $B$ vitamins & 0.49 & 0.52 \\
\hline 13. & Phrynoderma as a deficiency of essential fatty acid and vitamin E & $0 \cdot 27$ & $0.08^{*}$ & 28. & Food as major source of micronutrients for the bodyt & 0.58 & $0 \cdot 20$ \\
\hline 14. & Vitamin C deficiency as a cause for spongy bleeding gums & $0 \cdot 37$ & 0.44 & 29. & Fruits and vegetables as source of vitamins and minerals & $0 \cdot 86$ & $0 \cdot 16^{*}$ \\
\hline \multirow[t]{2}{*}{15.} & Vitamin C deficiency pre-disposing to infectionst & $0 \cdot 28$ & 0.24 & 30. & Cereals \& pulses as starch-rich foods & $0 \cdot 13$ & $0 \cdot 20$ \\
\hline & & & & 31. & Pulses as protein-rich foods & $0 \cdot 11$ & 0.24 \\
\hline
\end{tabular}

The item difficulty index $p$ is the ratio of the number of correct responses to the total number of responses $(n 92)$. The higher the index value, lower the difficulty. $D$ is the item discrimination index. *Indicates the items discarded due to low $D$.

+Indicates the items discarded due to low item validity index $(r)$ value of 0.0335 to 0.1319
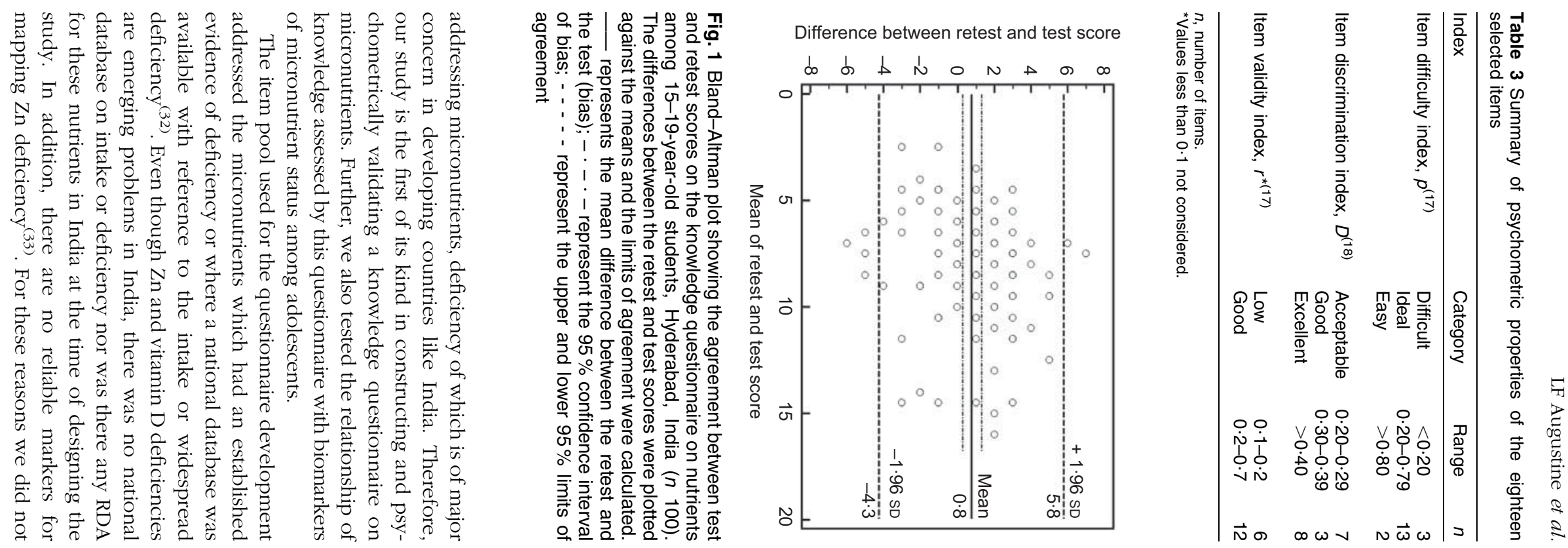
Table 4 Indicators of micronutrient status according to knowledge score below or above the 50th percentile: 15-19-year-old students, Hyderabad, India

\begin{tabular}{|c|c|c|c|c|c|}
\hline \multirow[b]{2}{*}{ Biomarker } & \multicolumn{2}{|c|}{ Below 50th percentile } & \multicolumn{2}{|c|}{ Above 50th percentile } & \multirow[b]{2}{*}{$P$ value } \\
\hline & Mean & SD & Mean & SD & \\
\hline $\mathrm{Hb} g / \mathrm{dl}$ & $13 \cdot 7$ & $2 \cdot 36$ & $14 \cdot 1$ & $2 \cdot 64$ & 0.346 \\
\hline Ascorbic acid (mg/dl) & 0.42 & 0.282 & $0 \cdot 42$ & $0 \cdot 308$ & $0 \cdot 758$ \\
\hline Retinol $(\mu \mathrm{g} / \mathrm{dl})$ & $24 \cdot 8$ & $6 \cdot 69$ & $28 \cdot 0$ & $7 \cdot 67$ & $0.022^{*}$ \\
\hline$\alpha$-Tocopherol $(\mu \mathrm{g} / \mathrm{dl})$ & 326 & $200 \cdot 0$ & 331 & $99 \cdot 5$ & 0.369 \\
\hline Folic acid $(\mathrm{ng} / \mathrm{ml})$ & $5 \cdot 4$ & $1 \cdot 84$ & $5 \cdot 6$ & 2.09 & $0 \cdot 418$ \\
\hline Vitamin $\mathrm{B}_{12}(\mathrm{pg} / \mathrm{ml})$ & 244 & $108 \cdot 1$ & 248 & $114 \cdot 9$ & 0.946 \\
\hline Ferritin $(\mathrm{ng} / \mathrm{ml}) \dagger$ & $46 \cdot 9$ & $101 \cdot 47$ & $40 \cdot 4$ & 35.06 & 0.758 \\
\hline
\end{tabular}

include $\mathrm{Zn}$ and vitamin $\mathrm{D}$ in the item pool. Iodine was not included on account of the universal salt iodization programme ${ }^{(34)}$ currently in operation in the country.

The selection of questions from the item pool was perhaps the most difficult step involved in our study. The expertise of the panel with regard to the domain of knowledge tested and the anticipated difficulty level of the item were considered while selecting questions. The nutrition component in the school curriculum, and nutrition messages propagated by the Government of India through the popular electronic media (television), were the basis for this. Accordingly, a number of questions on dietary sources that were judged to be inappropriate were deleted from the item pool. Questions related to nutrient absorption were also deleted as they were judged to be too technical for the group being studied. Care was also taken to select items which were simple, easily understandable and suitable for 15-19-year-old adolescent students of low and middle income level of economic status. Thus the selected thirty-one questions mostly attempted to test basic knowledge on micronutrients.

To obtain a valid questionnaire applying appropriate statistical methods, the thirty-one items were pre-tested in a group of adolescents. The statistical tools applied, such as item difficulty index, item discrimination index, item validity index and reliability test, provided an eighteenitem questionnaire. The spread of data, difficulty level and discriminative power of this eighteen-item questionnaire were within acceptable ranges. Nine items belonging to the domain of dietary sources did not show significant item to total correlation and were discarded. Some of the items had low inter-correlations but were retained due to content validity. The reliability of the questionnaire of $\alpha=0.71$ was also within the acceptable range and is in agreement with the widely accepted general nutrition knowledge test ${ }^{(3)}$ and a knowledge questionnaire developed for US children which had an $\alpha$ of $0 \cdot 7^{(12)}$. Thus we constructed a short self-administered questionnaire that required $15 \mathrm{~min}$ to complete with no gender-specific items and so can be used for adolescents.
This had an added advantage of easy assessment of knowledge useful in pre-post knowledge assessment studies on micronutrients.

In an attempt to understand whether true knowledge will be reflected in biomarkers of micronutrient status, we applied this questionnaire in a group of adolescent boys. The prevalence of anaemia among this study group was $21 \%$, which is similar to the anaemia prevalence among males (15-49 years) in the State of Andhra Pradesh as well as the national average ${ }^{(34)}$. Further, the prevalence of subclinical vitamin A deficiency of $17.5 \%$ and ascorbic acid deficiency of $75.0 \%$ reported in the present study were similar to those in a study among apparently healthy adults from western India which reported $21 \%$ and $77 \%$ respectively ${ }^{(35)}$. The other micronutrient deficiencies observed in the study population were similar to those reported in the literature in a similar group from a similar location $^{(36)}$, implying the representativeness of the study sample.

Among the micronutrients tested, plasma retinol alone showed a positive relationship with knowledge score, suggesting that knowledge on micronutrients can influence the status of at least some nutrients. This relationship persisted after controlling for economic status, a known confounder for micronutrient status on account of affordability and thereby intake ${ }^{(37)}$.

Evidence relating knowledge, dietary intake and biomarkers is meagre except for one study among nineteen volunteers that evaluated the impact of dietary counselling among cancer survivors, with an experimental group receiving dietary counselling to increase fruit and vegetable consumption. The plasma levels tested were for carotenoids, vitamin C, $\alpha$-tocopherol and cholesterol. Plasma carotenoid levels and ascorbic acid showed a change with acceptance and compliance to diet therapy ${ }^{(38)}$. Another study from western India, where population characteristics were similar to our study group, examined the relationship between dietary habits, plasma retinol and plasma ascorbic acid among 214 men and 108 women. Results showed a dose-response relationship of intake with 
serum retinol alone and not with vitamin $\mathrm{C}^{(35)}$. The findings were explained on the basis that vitamin $\mathrm{A}$ is fat soluble, can be stored in the body and therefore better reflects the dietary habits of the individual. The present study findings can also be explained along similar lines, with knowledge influencing dietary habits which are reflected in biomarker levels. Data on fat intake were not collected. However, the study in western India was done in a population where socio-economic status was similar to our study participants, and it is believed that fat intake would also be similar in both groups. In the absence of any other reported literature, it would not be wrong to consider retinol status as a good biomarker sensitive to knowledge on micronutrients and which may change with changes in knowledge following dietary advice.

The implications of our study findings are varied. First, they emphasize the need to use validated questionnaires in nutrition research involving qualitative aspects like knowledge. Second, they show that knowledge on micronutrients, if assessed correctly, may relate to the blood levels of specific micronutrients of the target group as reported herein for plasma retinol. Increasing the knowledge of the population increases the sustainability of interventions as well as voluntary compliance by the target population.

Although parental factors can influence food choices, the participants of the present study were close to adulthood and therefore culturally possessed a certain amount of autonomy in their food choices. The majority of the participants had a higher educational qualification than their parents and contributed in performing family food chores. This background information on the study participants gives us sufficient confidence in using them as 'change agents'.

There are limitations that need to be considered when interpreting the study results. The generalizability of the test-retest data may be limited since the study was done in girls alone, even though the pre-test showed no gender difference in scoring. Dietary intake data were not collected, which could have provided more conclusive evidence for the relationship we found. A positive relationship of knowledge with micronutrients like folic acid (where body stores are not available) cannot be ruled out, on account of the time lag of 6 months between the assessments of micronutrient status and knowledge.

\section{Acknowledgements}

The authors wish to acknowledge the funding received from the Indian Council of Medical Research for carrying out the study. The Fellowship received by L.F.A. from the University Grants Commission, Government of India, is also acknowledged. The authors declare that there is no conflict of interest. All authors have participated in the research reported and all have agreed to be an author of this paper. K.M.N. conceptualized and designed the study, supervised the study protocol and edited the manuscript. S.V. conceptualized the psychometric validation of the test scale, was involved in development of the questionnaire at various stages and edited the manuscript. L.F.A. was responsible for designing the questionnaire, conduct of studies, analysis of ferritin and has written the manuscript. A.L. did clinical examination for the project and applied inclusion/exclusion criteria. M.V.R. was responsible for data analysis. S.F.R. was responsible for application of the validated scale in the study on 'Stress, allostatic load and micronutrient status among students: impact of dietary advice' and was involved in interpreting the test scales. P.R. analysed biomarkers of micronutrient status and V.V.R. analysed plasma retinol and $\alpha$-tocopherol. All authors have read and contributed to the final manuscript. The authors thank the Nutrition Society of India for the opportunity to present part of the results of the study at its 42nd Annual Conference held in Mumbai, India, 19-20 November 2010. They also thank Dr B. Sesikeran MD, Director, National Institute of Nutrition, for his support and encouragement.

\section{References}

1. Vijayaraghavan K (2002) Control of micronutrient deficiencies in India; obstacles and strategies. Nutr Rev 60, 5 Pt 2, S73-S76.

2. Kline P (1993) The Handbook of Psychological Testing. London: Routledge.

3. Parmenter K \& Wardle J (1999) Development of a general nutrition knowledge questionnaire for adults. Eur J Clin Nutr 53, 298-308.

4. Hendrie GA, Cox DN \& Coveney J (2008) Validation of the general nutrition knowledge questionnaire in an Australian community sample. Nutr Diet 65, 72-77.

5. Shaaban SY, Nassar MF, Abd Elhamid DM et al. (2009) Nutritional knowledge and attitude of adolescent school girls living in Cairo. Res J Med Med Sci 4, 421-427.

6. Turconi G, Celsa M, Rezzani C et al. (2003) Reliability of a dietary questionnaire on food habits, eating behaviour and nutritional knowledge of adolescents. Eur J Clin Nutr 57, $753-763$.

7. Anderson AS, Bell A, Adamson A et al. (2001) A questionnaire assessment of nutrition knowledge - validity and reliability issues. Public Health Nutr 5, 497-503.

8. Lambert LG, Monroe A \& Wolff L (2010) Mississippi elementary school teachers' perspectives on providing nutrition competencies under the framework of their school wellness policy. J Nutr Educ Behav 42, 271-276.

9. Zarnowiecki D, Dollman J \& Sinn N (2011) A tool for assessing healthy food knowledge in 5-6-year-old Australian children. Public Health Nutr 14, 1177-1183.

10. Resnicow K, Hearn M, Delano RK et al. (1997) Development of a nutrition knowledge scale for elementary school students: toward a national surveillance system. J Nutr Educ 28, 156-164.

11. Steenhuis IHM, Brug J, Van Assema P et al. (1996) The validation of a test to measure knowledge about the fat content of food products. Nutr Health 10, 331-339.

12. Stevens J, Cornell CE, Story M et al. (1999) Development of a questionnaire to assess knowledge, attitudes, and behaviors in American Indian children. Am J Clin Nutr 69 , 4 Suppl., 773S-781S. 
13. O'Brien G \& Davies M (2007) Nutrition knowledge and body mass index. Health Educ Res 22, 571-575.

14. Nuss H, Freeland-Graves J, Clarke K et al. (2007) Greater nutrition knowledge is associated with lower 1-year postpartum weight retention in low-income women. $J \mathrm{Am}$ Diet Assoc 107, 1801-1806.

15. Worsley A (2002) Nutrition knowledge and food consumption: can nutrition knowledge change food behaviour? Asia Pacific J Clin Nutr 11, Suppl. 3, S579-S585.

16. Planning Commission (2002-2007) Report of the Sub-group on Adolescents for the Tenth Five Year Plan. New Delhi: Planning Commission, Government of India; available at http://planningcommission.nic.in/aboutus/committee/wrkgrp/ wg_adolcnts.pdf

17. Garret HE \& Woodworth RS (1969) The reliability and validity of test scores. In Statistics In Psychology and Education, pp. 337-370. New York: David McKay Company Inc.

18. Ebel RL (1972) Essentials of Educational Measurement, 1st ed. Englewood Cliffs, NJ: Prentice Hall.

19. Wilson AM, Magarey AM \& Mastersson N (2008) Reliability and relative validity of a child nutrition questionnaire to simultaneously assess dietary patterns associated with positive energy balance and food behaviours, attitudes, knowledge and environments associated with healthy eating. Int J Behav Nutr Phys Act 5, 5.

20. Myles PS \& Cui J (2007) Using the Bland-Altman method to measure agreement with repeated measures. Br J Anaesth 99, 309-311.

21. Rosner B (2006) Multisample inference. In Fundamentals of Biostatistics, 6th ed., pp. 568-571. Belmont, CA: Duxbury Thomson-Brooks/Cole.

22. International Nutritional Anaemia Consultative Group (1985) Measurement of Iron Status. A Report of the International Nutritional Anaemia Consultative Group (INACG), pp. 4-8. Washington, DC: The Nutrition Foundation Inc.

23. Pawashe AB, Raman L, Nair M et al. (1987) Validity of using capillary blood for the measurement of plasma ferritin. Clin Chim Acta 163, 119-120.

24. Bieri JG, Tolliver TJ \& Catignani GL (1979) Simultaneous determination of $\alpha$-tocopherol and retinol in plasma or red cells by high pressure liquid chromatography. Am J Clin Nutr 32, 2143-2149.

25. Zannoni V, Lynch M \& Goldstein S (1974) A rapid micromethod for the determination of ascorbic acid in plasma and tissues. Biochem Med 11, 41-48.
26. Young DS (1987) Implementation of SI units for clinical laboratory data, style specifications and conversion tables. Ann Intern Med 106, 114-129.

27. International Institute for Population Sciences \& ORC Macro (2000) National Family and Health Survey (NFHS-2). Mumbai: IIPS.

28. Lakshman RR, Sharp SJ, Ong KK et al. (2010) A novel school-based intervention to improve nutrition knowledge in children: cluster randomised controlled trial. BMC Public Health 10, 123.

29. Lanerolle P \& Atukorala S (2006) Nutrition education improves serum retinol concentration among adolescent school girls. Asia Pac J Clin Nutr 15, 43-49.

30. Joshi N \& Vijayalaxmi KG (2009) Nutritional education tool to improve overall dietary attitude and knowledge among young women. J Hum Ecol 25, 187-191.

31. Nazni P \& Vimala S (2010) Nutrition knowledge, attitude and practice of college sportsmen. Asian J Sports Med 1, 93-100.

32. National Nutrition Monitoring Bureau (2003) Prevalence of Micronutrient Deficiencies. NNMB Technical Report Series no. 21. Hyderabad: National Institute of Nutrition; available at http://www.nnmbindia.org/NNMB\%20MND \%20REPORT\%202004-Web.pdf

33. International Zinc Nutrition Consultative Group, Brown $\mathrm{KH}$, Rivera JA et al. (2004) International Zinc Nutrition Consultative Group (IZiNCG) technical document \#1. Assessment of the risk of zinc deficiency in populations. Food Nutr Bull 25, 1 Suppl. 2, S130-S161.

34. International Institute for Population Sciences \& ORC Macro (2007) National Family and Health Survey (NFHS-3). Mumbai: IIPS.

35. Chiplonkar SA, Agte VV, Mengale SS et al. (2002) Are lifestyle factors good predictors of retinol and vitamin C deficiency in apparently healthy adults? Eur J Clin Nutr 56, 96-104.

36. Sivakumar B, Nair KM, Sreeramulu D et al. (2006) Effect of micronutrient supplement on health and nutritional status of school children: biochemical status. Nutrition 22, 1 Suppl., S15-S25.

37. Monsivais P, Aggarwal A \& Drewnowski A (2010) Low micronutrient intake is associated with low socio-economic status and low diet cost. FASEB J 24, lb 359 .

38. Le Marchand L, Hankin JH, Carter FS et al. (1994) A pilot study on the use of plasma carotenoids and ascorbic acid as markers of compliance to a high fruit and vegetable dietary intervention. Cancer Epidemiol Biomarkers Prev 3, 245-251. 